\title{
Evaluation of anti-cancer activity of Brinzolamide with special emphasis on blood cancer (Polycethemia Rubra Vera) in vivo
}

\author{
Keval R. Rathod ${ }^{1 \star}$, Tejas H. Ganatra ${ }^{1}$ \\ 'Department of Pharmacology, School of Pharmacy, R K University, Kasturbadham, \\ Rajkot, Gujarat, India
}

\begin{abstract}
:
Introduction: Polycythemia is an uncommon type of cancer in which bone marrow produce too many red blood cells as well as due to overproduction of white blood cells and platelets. It is caused by neoplastic proliferation and maturation of erythroid, Megakaryocytic and Granulocytic elements to produce Panmyelosis. Looking at the dire to find better \& safer alternate, we decided to work on Brinzolamide.

Methodology: In vivo anti-cancer potential of brinzolamide was evaluated using Cobalt containing Milk induced polycythemia rubra vera (PRV) on Swiss albino rat. Animals were subjected for 30 days treatment of Co-Milk along with respective drug administration. In this model, Methotrexate was taken as standard because of structural \& mechanism similarity with brinzolamide.

Result: In Cobalt containing milk induced PRV, there was significant reduction in all the blood cell counts, increased blood flow rate \& shown beneficial effects on other relevant parameters as well as shown protective action on spleen.

Conclusion: From the study conducted, we can conclude that Brinzolamide can serve as one of the alternate for blood cancer treatment specifically in Polycythemia rubra vera.

Keywords: Brinzolamide, Cobalt containing Milk induced Polycythemia, cytotoxicity, Spleenomegaly.
\end{abstract}

\section{Introduction:}

Blood cancer affect the production and function of Human blood cells. Most of these cancer start in bone marrow where blood is produced. Stem cells presence in bone marrow mature and develop into three types of blood cells: Red blood cells, White blood cells and Platelets. In most blood cancers, the normal blood cell development process is interrupted by uncontrolled growth of an abnormal type of blood cell. These abnormal blood cells, prevent blood from performing many of its functions, like fighting of infections or preventing serious bleeding. There are three main types of blood cancers:

(i) Leukemia, a type of cancer found in blood and bone marrow is caused by the rapid production of abnormal White blood cells and it is a cancer that originates in blood forming tissue. ${ }^{[1]}$ 
(ii) Lymphoma, is a type of blood cancer that affects the lymphatic system, which removes excess fluids from body and produces immune cells.

(iii) Myeloma, is a cancer of the plasma cells. Plasma cells are white blood cells are white blood cells that disease and infection, fighting antibodies in human body. Myeloma cells prevents the normal production of antibodies, leaving our body's immune system weekend and susceptible to infection. ${ }^{[2]}$

Polycythemia Vera (PV), essential thrombocythemia (ET) and myeloid metaplasia with myelofibrosis (MMM) are clonal disorders arising from hematopoietic progenitors. An internet based protocol for clinical information and biological specimens suggest that a recurrent somatic missense mutation JAK2V617F in granulocyte DNA. Molecular and cytogenetic analysis demonstrated that this mutation were due to duplication of the mutant allele. Treatment for blood cancer depends on the type of cancer, your age, how fast the cancer is progressing, where the cancer has spread and other factors, some common blood cancer treatment include: Stem cell Transplantation, Chemotherapy, Radiation Therapy. ${ }^{[3-13]}$

Because of the need of this treatment we found some alternative approach to satisfy this need and it is Brinzolamide. Brinzolamide is a sulfa drug and its structure contain sulfonamide moiety. Rationale: Carbonic Anhydrase II is an important enzyme for the human body playing key role in regulation of apoptosis. ${ }^{[14]}$ Literatures revels that Carbonic Anhydrase Inhibitors is induce the apoptosis of the cell and also reduce the cancer cell proliferation. ${ }^{[14]}$ Apart from this, it is well reported that CA II level is raised in myeloid tissues and CA II inhibitors can play significant role in inhibition of progression of myeloid tissue. ${ }^{[16]}$ Sulfonamide are having anti-cancer potential and can inhibit progression of Cancer. ${ }^{[17]}$ As Brinzolamide is CA II inhibitor, belonging to Sulfonamide category ${ }^{[17]}$, based on above mentioned rationale we planned to evaluate anti cancer potential of the same with different experimental models with special emphasis on Polycythemia rubra vera.

\section{Material and Methodology:}

\section{Procurement of Brinzolamide:}

We procured a brinzolamide as drug of ophthalmic suspension from the market of Rajkot as the brand name of Brinolar.

\section{Evaluation of Anti-cancer potential of Brinzolamide using Animal model: ${ }^{[18]}$}

The study was involving use of albino Wistar rat and prior permission from IAEC was taken. Approved Research protocol number was RKCP/COL/RP/18/84.

In this model, we induced the polycythemia Vera in Rat using Cobalt Chloride $\left(\mathrm{CoCl}_{2}\right)$. In this procedure, we gave the basal diet of whole milk with $0.5 \mathrm{mg}$ iron and $0.025 \mathrm{mg}$ of copper daily with $0.5 \mathrm{mg}$ of Cobalt Chloride $\left(\mathrm{CoCl}_{2}\right)$.

As per some literature review Methotrexate is a general drug for cancer and give effective result on Polycythaemia Vera. ${ }^{[19]}$ 


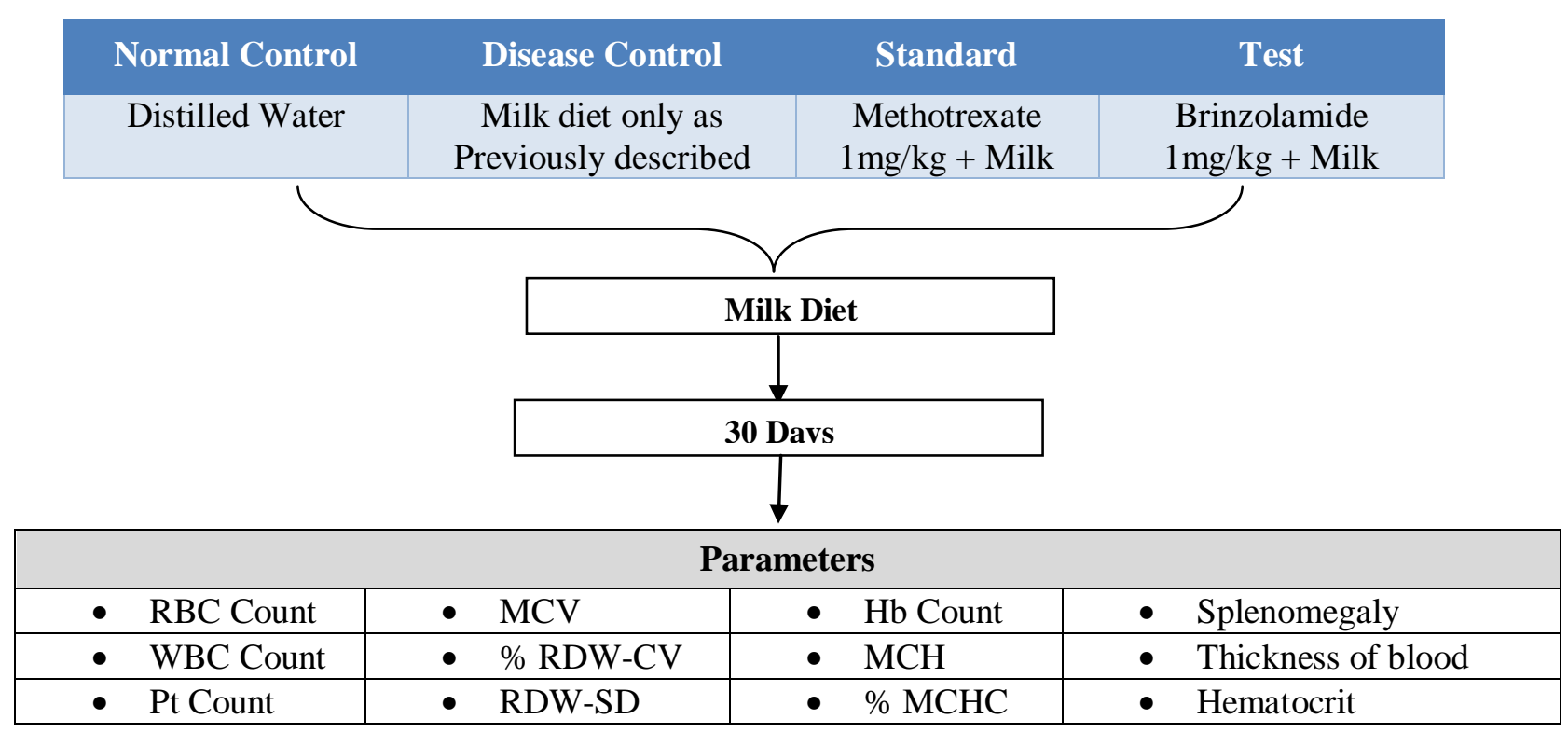

\section{Whole Blood Parameters:}

We measured all the blood parameters using Blood cell counter. We are having blood cell counter of Merilyzer celquant 3s brand.

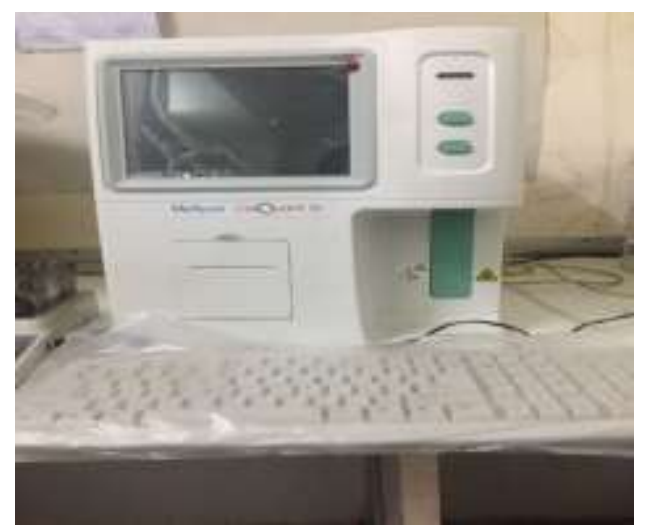

Fig 1 Meilyzer CelQuant

\section{Splenomegaly Study:}

The spleen is about the same color as the liver and is attached to the greater curvature of the stomach.

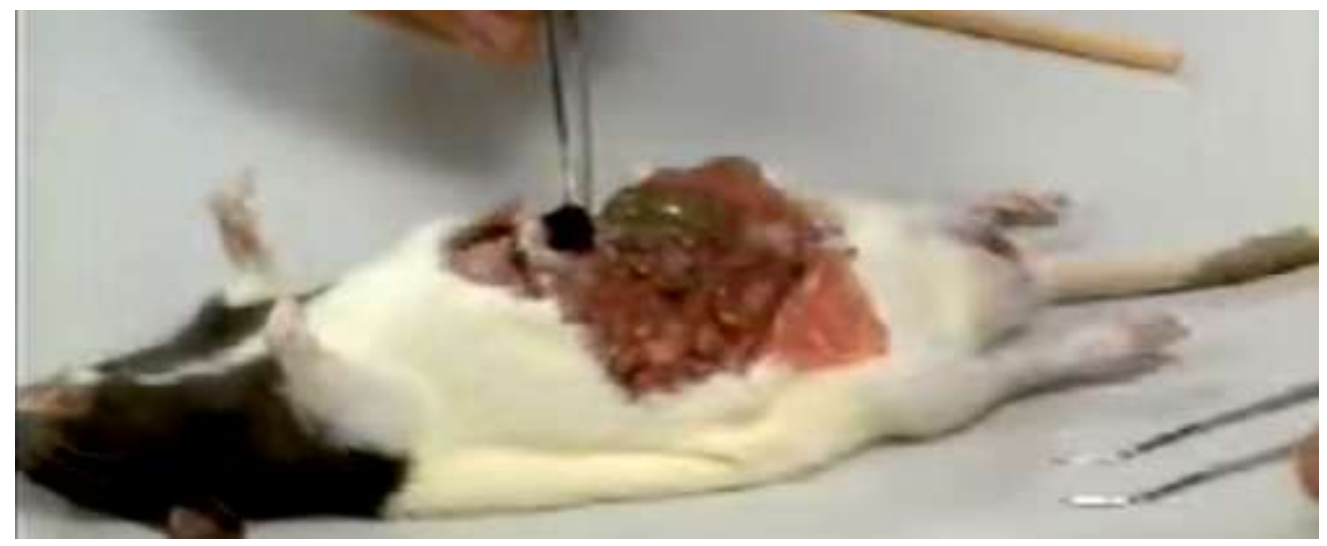

Fig 2 Location of spleen in Dissected Rat 
It is attached with the circulatory system and functions in the destruction of the blood cells and blood storage. ${ }^{[23]}$ In this model we dissect the animal and take the spleen outside and then measure the size of the spleen of different groups of animal. ${ }^{[20]}$

\section{Blood Thickening Study:}

The important parameter is the blood is being thicker in this condition. So for this purpose we collect some blood into small capillary so literally the blood is block into the capillary. ${ }^{[21]}$ The time required to block the capillary was recorded \& compared among different groups.

\section{Statistical Analysis:}

Statistical analysis of results was done by ANOVA test followed by turkey's test for determination of variance. Data were considered significantly different from each other if $p \leq 0.05$ and if $p \leq 0.001$ then the difference between data were consider highly significant.

\section{Result:}

Effect on RBC (Red Blood Cells) count in Cobalt containing milk Inducing Polycythemia Rubra Vera:

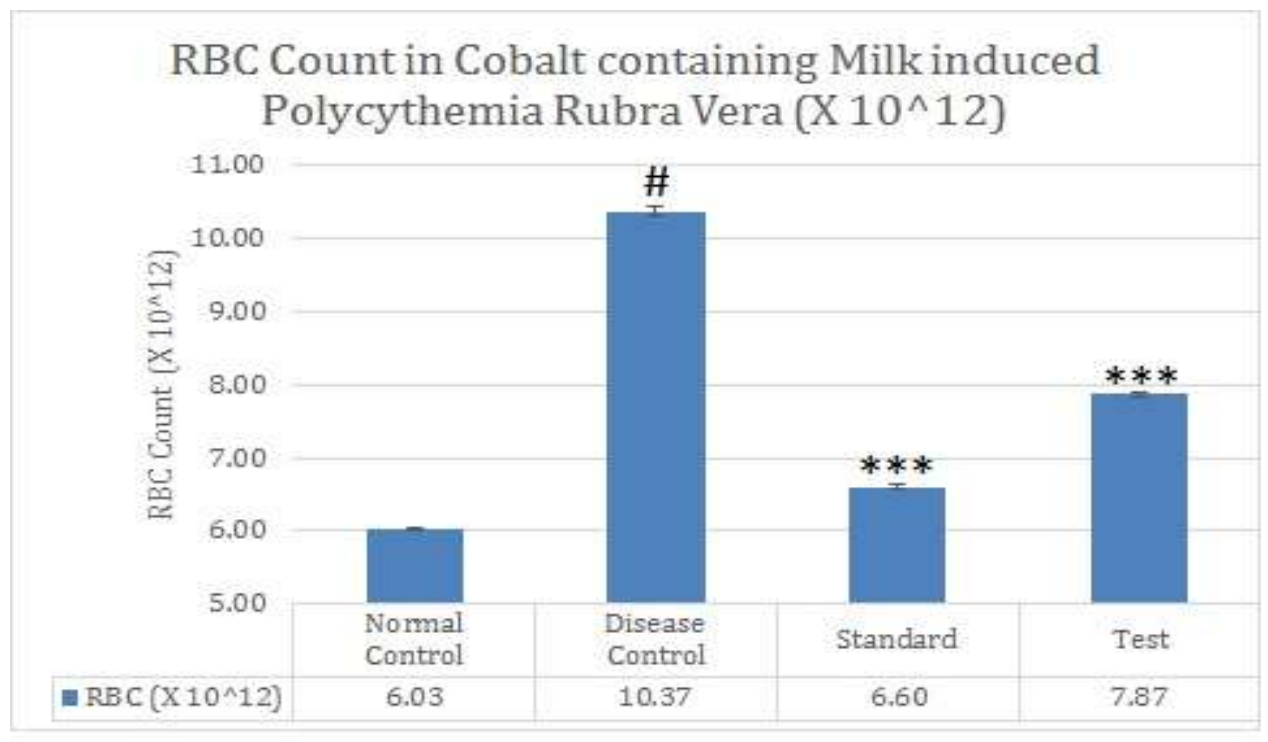

Fig 2 Effect on RBC count in Cobalt containing milk Inducing Polycythemia rubra Vera

The finding of this model suggest that there was significant increase in RBC (X 10^12) count in Disease control $(9.37 \pm 0.065)$ compared to that of Normal control $(8.02 \pm 0.021)$. While it was found significantly lesser in Standard (6.6 \pm 0.029$)$ and Test $(7.87 \pm 0.032)$ compared to that of Disease control. 


\section{Effect on WBC (White Blood Cells) count in Cobalt containing milk Inducing Polycythemia} Rubra Vera:

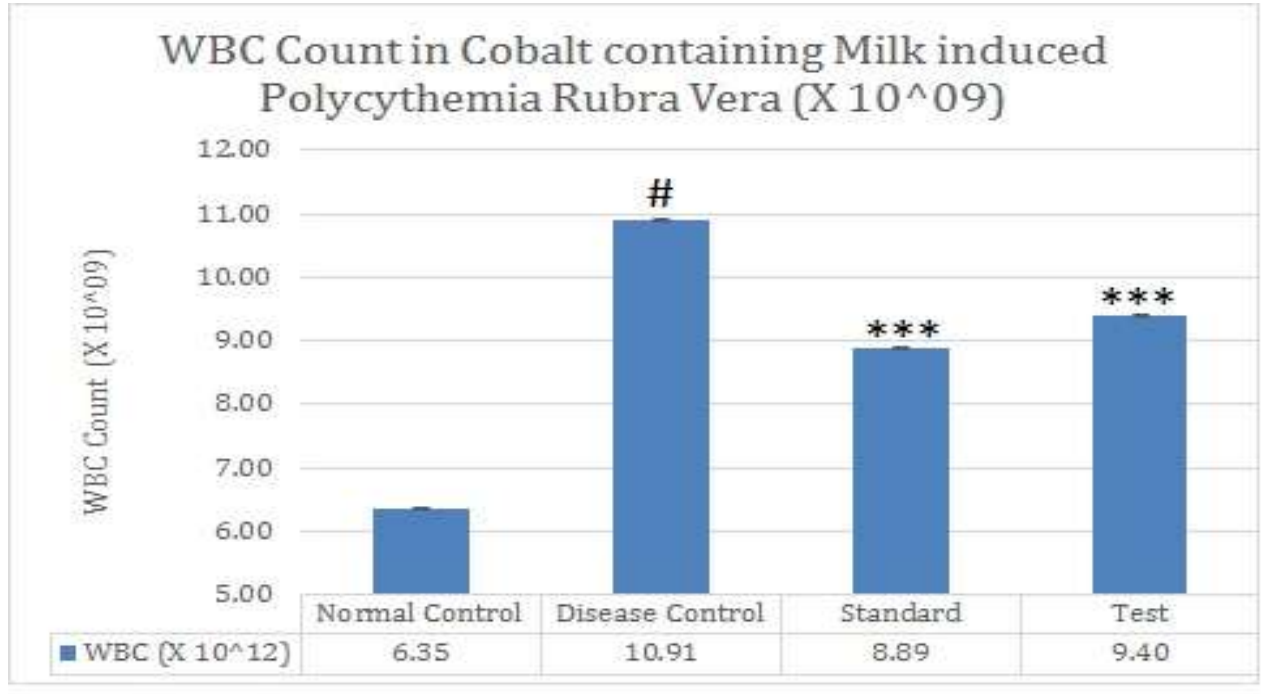

Fig 3 Effect on WBC count in Cobalt containing milk Inducing Polycythemia rubra Vera

The finding of this model suggest that there was significant increase in WBC (X 10^09) count in Disease control (6.94 \pm 0.02$)$ compared to that of Normal control (5.35 \pm 0.54$)$. While it was found significantly lesser in

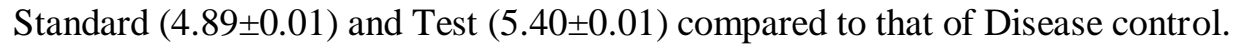

Effect on Platelets count in Cobalt containing milk Inducing Polycythemia Rubra Vera:

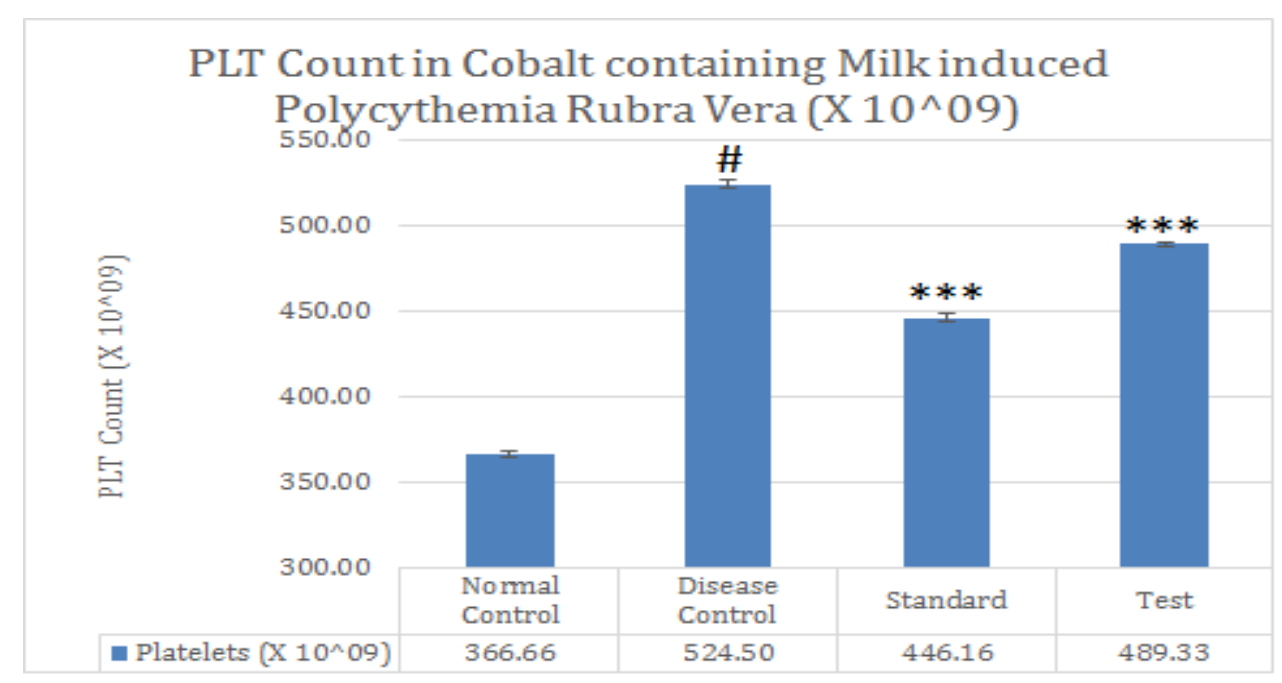

Fig 4 Effect on PLT count in Cobalt containing milk Inducing Polycythemia rubra Vera

The finding of this model suggest that there was significant increase in PLT (X 10^09) count in Disease control $(524.50 \pm 2.43)$ compared to that of Normal control $(366.66 \pm 1.70)$. While it was found significantly lesser in Standard $(446.16 \pm 2.68)$ and Test $(489.33 \pm 0.88)$ compared to that of Disease control. 
Effect on Hemoglobin count in Cobalt containing milk Inducing Polycythemia Rubra Vera:

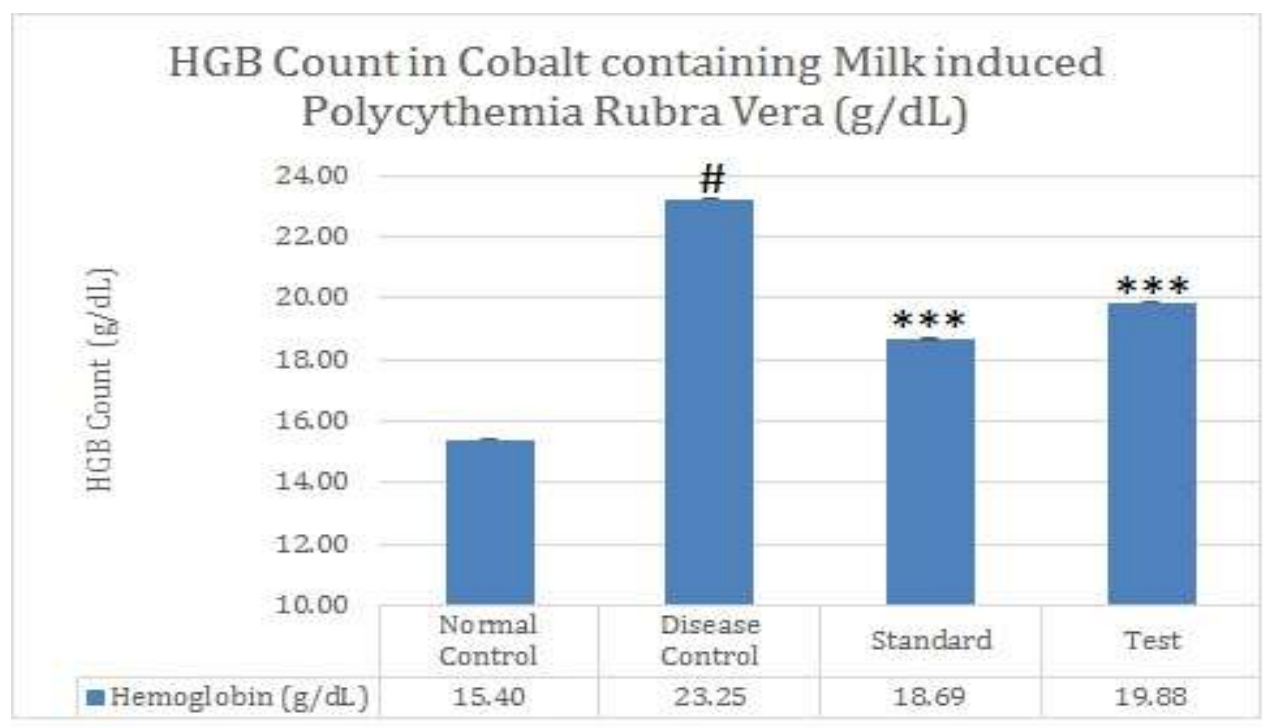

Fig 5 Effect on HGB count in Cobalt containing milk Inducing Polycythemia rubra Vera

The finding of this model suggest that there was significant increase in HGB $(\mathrm{g} / \mathrm{dL})$ count in Disease control

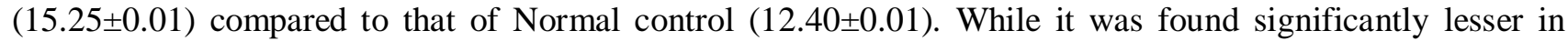

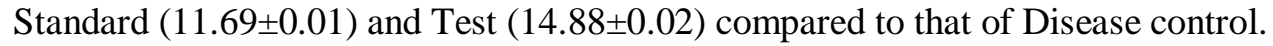

Effect on MCHC (Mean Corpuscular Hemoglobin Concentration - g/dL) in Cobalt containing milk Inducing Polycythemia Rubra Vera:

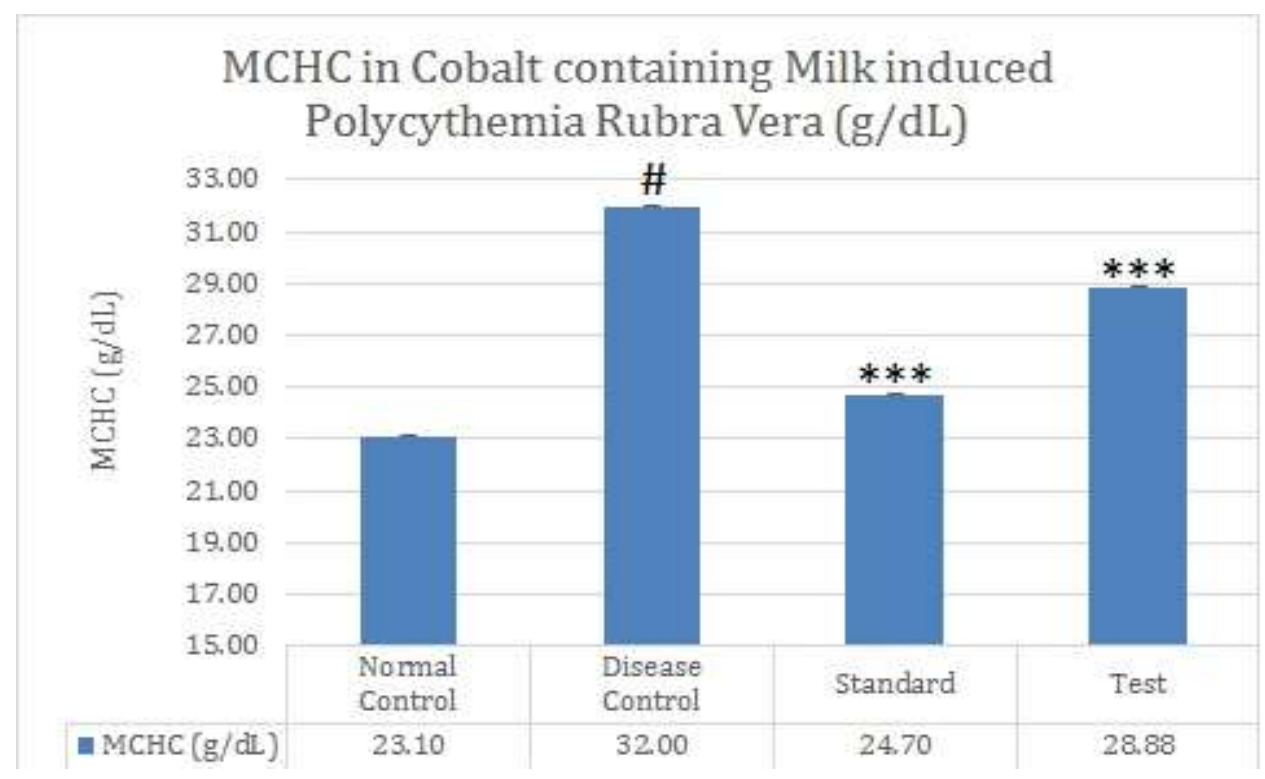

Fig 6 Effect on MCHC in Cobalt containing milk Inducing Polycythemia rubra Vera

The finding of this model suggest that there was significant increase in MCHC (g/dL) count in Disease control (30.80 \pm 0.01$)$ compared to that of Normal control $(23.10 \pm 0.01)$. While it was found significantly lesser in Standard (24.70 \pm 0.01$)$ and Test $(28.88 \pm 0.02)$ compared to that of Disease control. 
Effect on MCH (Mean Corpuscular Hemoglobin) Value in Cobalt containing milk Inducing Polycythemia Rubra Vera:

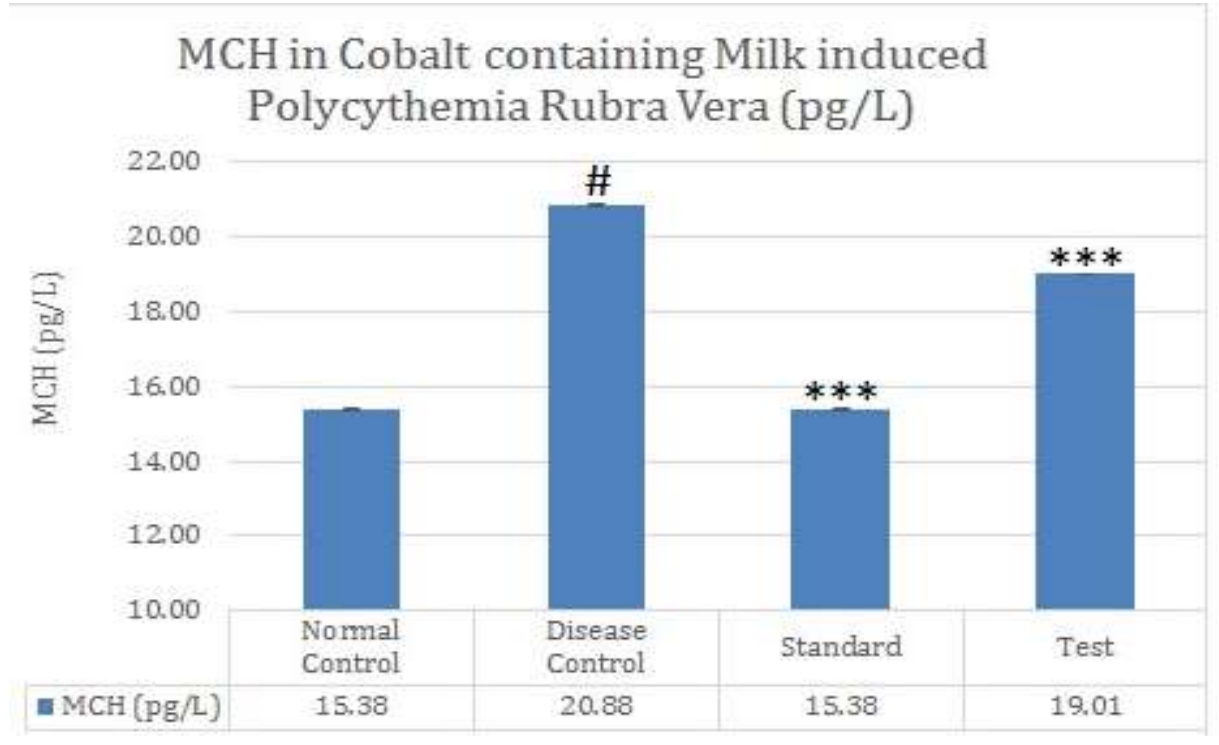

Fig 7 Effect on MCH Value in Cobalt containing milk Inducing Polycythemia rubra Vera

The finding of this model suggest that there was significant increase in $\mathrm{MCH}(\mathrm{pg} / \mathrm{L})$ count in Disease control (20.88 \pm 0.02$)$ compared to that of Normal control (15.38 \pm 0.02$)$. While it was found significantly lesser in Standard (15.38 \pm 0.02$)$ and Test (19.01 \pm 0.00$)$ compared to that of Disease control.

Effect on MCV (Mean Corpuscular Volume) Range in Cobalt containing milk Inducing Polycythemia Rubra Vera:

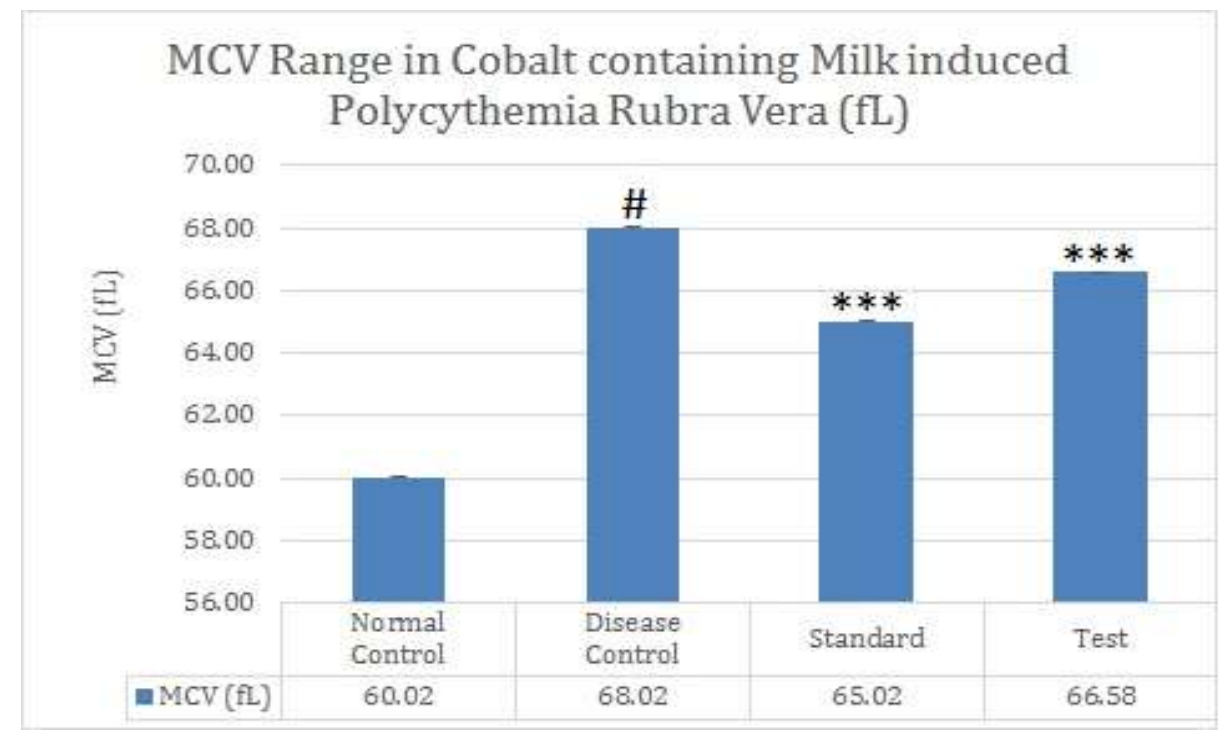

Fig 8 Effect on MCV Range in Cobalt containing milk Inducing Polycythemia rubra Vera

The finding of this model suggest that there was significant increase in MCV (fL) count in Disease control (68.02 \pm 0.00$)$ compared to that of Normal control $(60.02 \pm 0.01)$. While it was found significantly lesser in Standard (65.02 \pm 0.00$)$ and Test $(66.58 \pm 0.02)$ compared to that of Disease control. 
Effect on \% RDW-CV (Red Blood Cells Distribution Width) in Cobalt containing milk Inducing Polycythemia Rubra Vera:

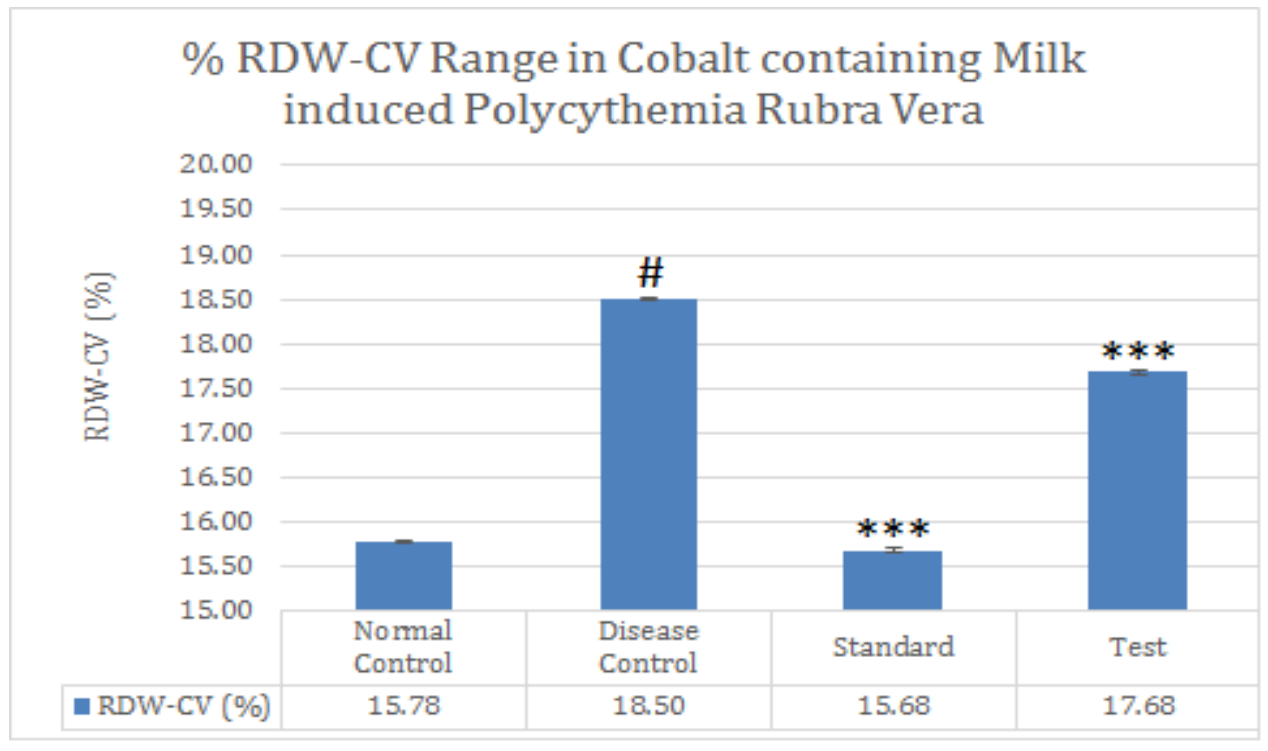

Fig 9 Effect on \% RDW-CV in Cobalt containing milk Inducing Polycythemia rubra vera

The finding of this model suggest that there was significant increase in RDW-CV (\%) count in Disease control $(16.01 \pm 0.00)$ compared to that of Normal control $(14.78 \pm 0.02)$. While it was found significantly lesser in Standard (15.68 \pm 0.02$)$ and Test (14.68 \pm 0.02$)$ compared to that of Disease control.

Effect on RDW-SD (Red Blood Cells Distribution Width - fL) in Cobalt containing milk Inducing Polycythemia Rubra Vera:

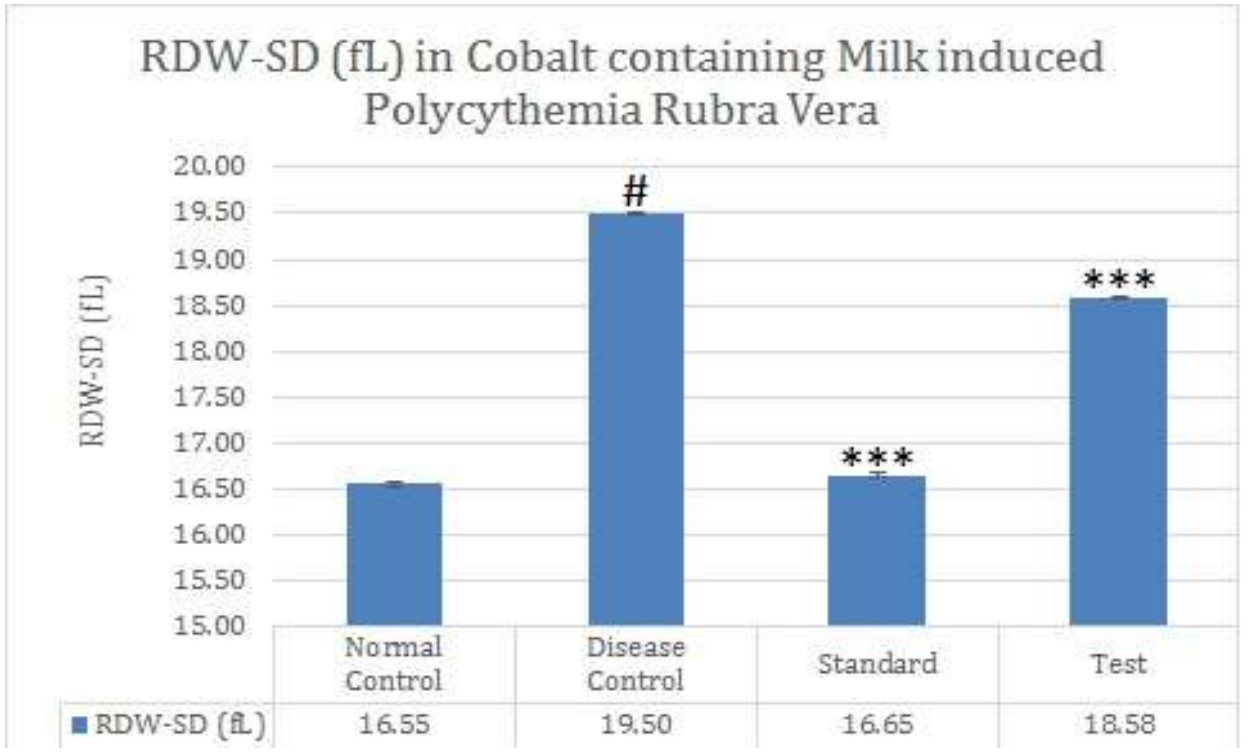

Fig 10 Effect on RDW-SD (fL) in Cobalt containing milk Inducing Polycythemia Rubra Vera

The finding of this model suggest that there was significant increase in RDW-SD (fL) count in Disease control (36.78 \pm 0.02$)$ compared to that of Normal control (33.38 \pm 0.02$)$. While it was found significantly lesser in Standard (32.38 \pm 0.02$)$ and Test (34.58 \pm 0.02$)$ compared to that of Disease control. 


\section{Effect on HCT (Hematocrit \%) in Cobalt containing milk Inducing Polycythemia Rubra Vera:}

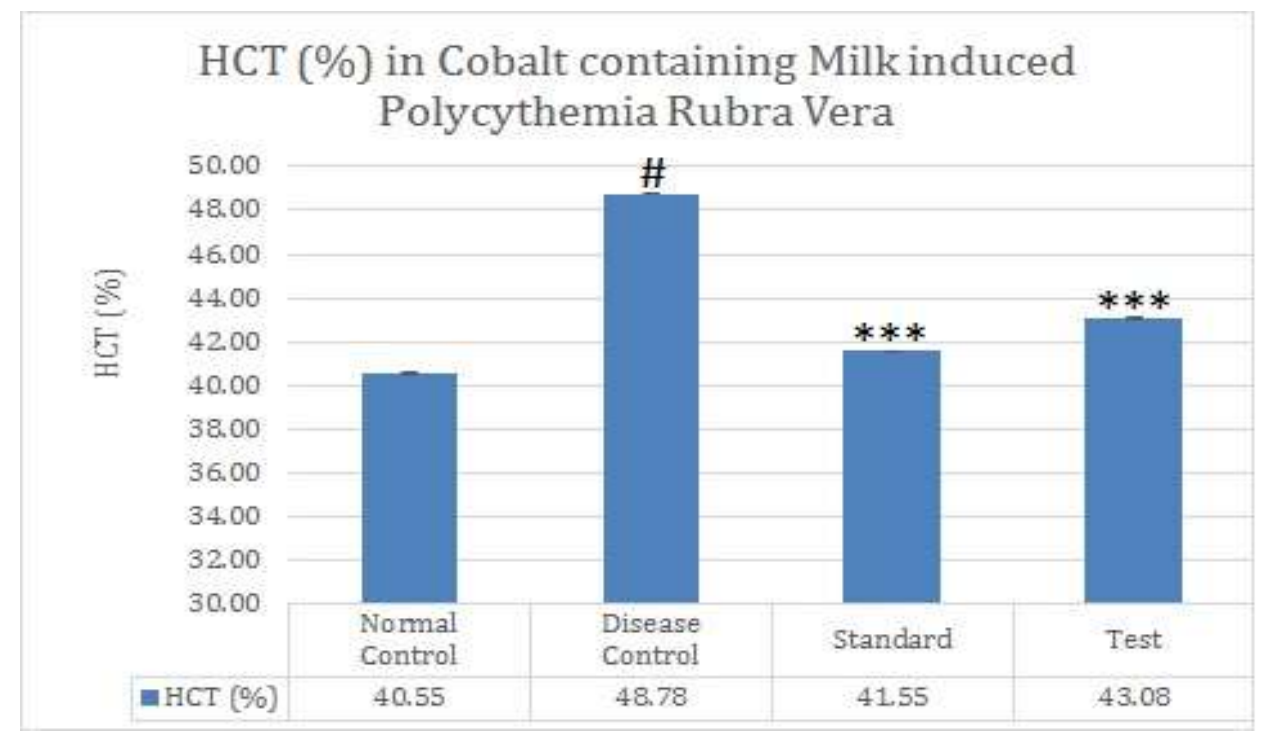

Fig 11 Effect on \% HCT in Cobalt containing milk Inducing Polycythemia Rubra Vera

The finding of this model suggest that there was significant increase in HCT (\%) count in Disease control (58.78 \pm 0.02$)$ compared to that of Normal control $(56.58 \pm 0.02)$. While it was found significantly lesser in

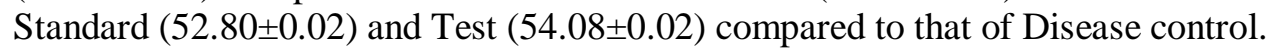

\section{Effect on Size and Weight of Spleen in Cobalt containing milk Inducing Polycythemia Rubra Vera:}

In this model, the animal were dissected and spleen was isolated. The size of the spleen was found as follow:

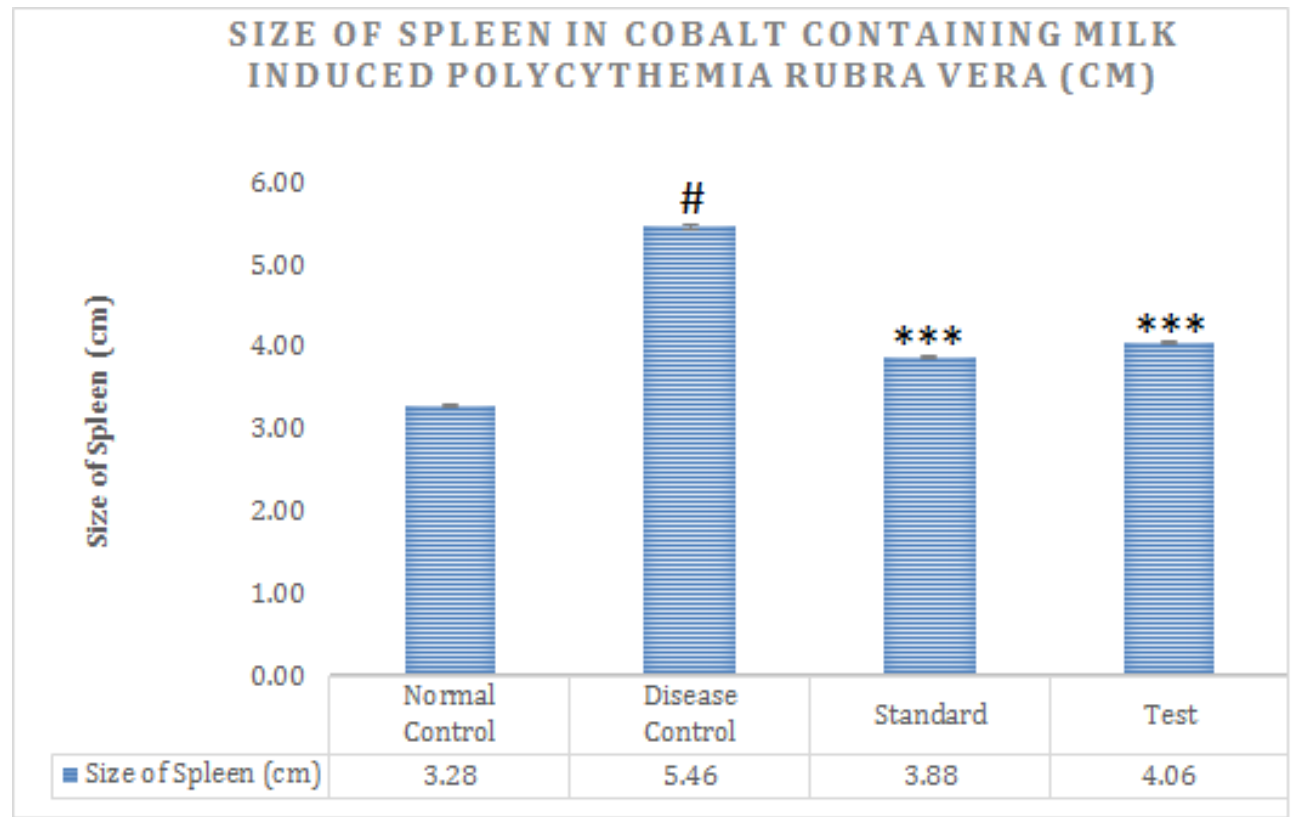

Fig 12 (A) Effect on size of Spleen in Cobalt containing milk Inducing Polycythemia Rubra Vera 
The study shown that there was significant increase in size of spleen in Disease control group (5.46 \pm 0.02 $\mathrm{cm})$ as compare to that of Normal control group $(3.28 \pm 0.02 \mathrm{~cm})$ which was found significantly lower in Standard control group $(3.88 \pm 0.02 \mathrm{~cm})$ and Test control $(4.06 \pm 0.02 \mathrm{~cm})$ compare to that of the Disease control group.

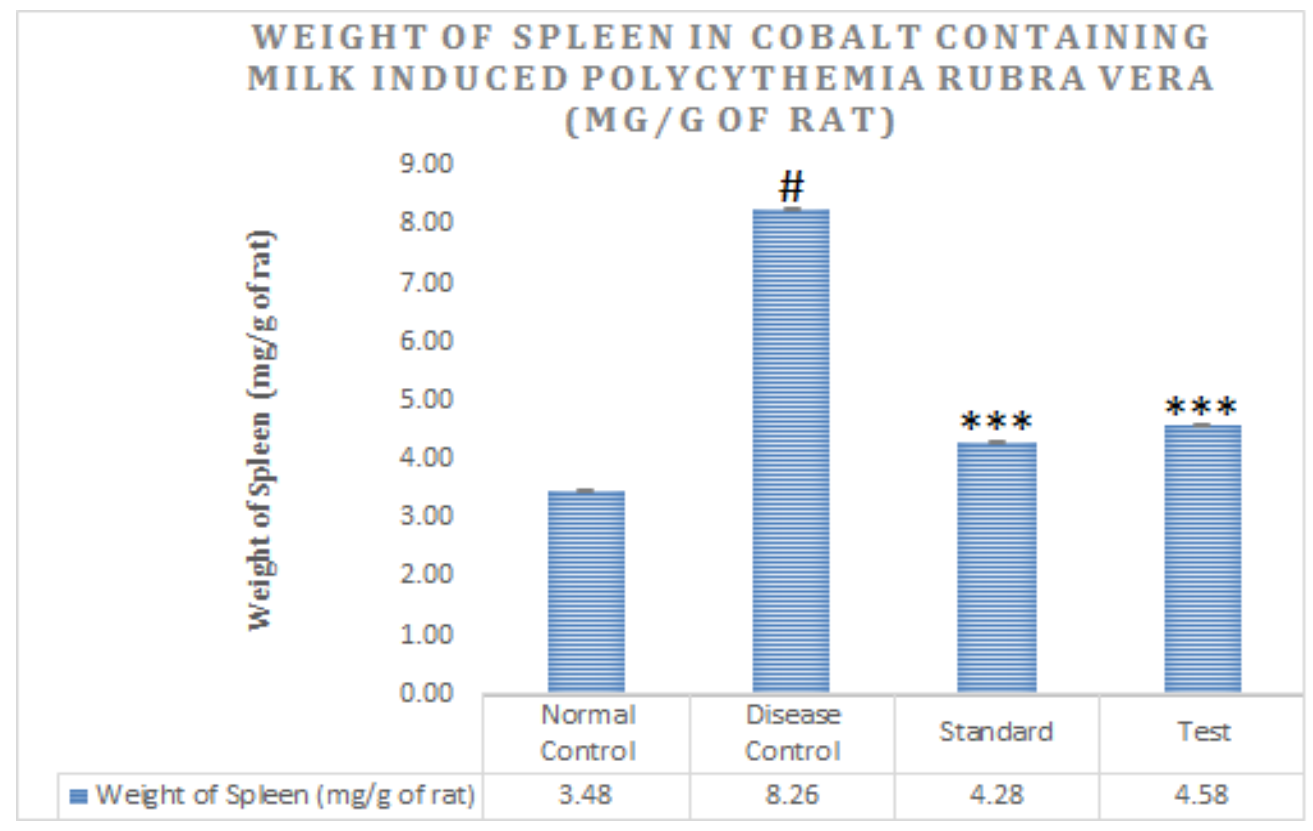

Fig 12 (B) Effect on weight of Spleen in Cobalt containing milk Inducing Polycythemia Rubra Vera

The study also shown that there was significant increase in weight of spleen in Disease control group ( $8.26 \pm$ $0.02)$ as compare to that of Normal control group $(3.48 \pm 0.02)$ which was found significantly lower in Standard control group $(4.28 \pm 0.02)$ and Test control $(4.58 \pm 0.02)$ h to that of the Disease control group.

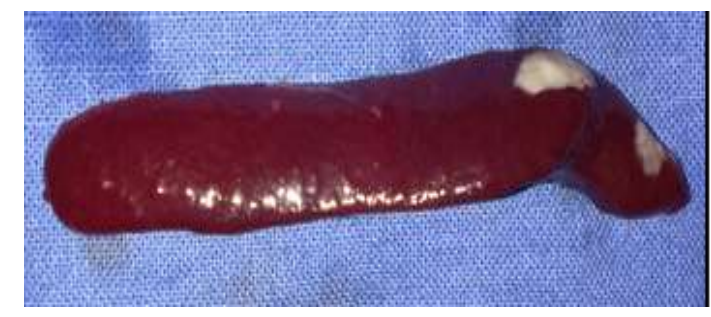

Normal Control group

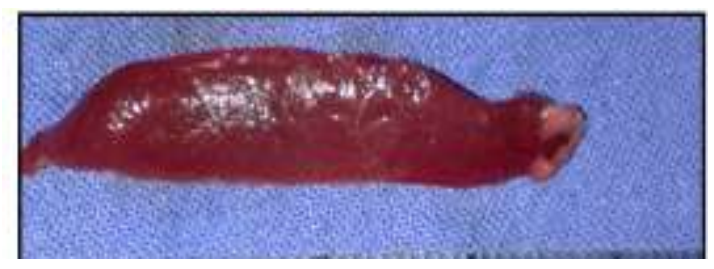

Standard group

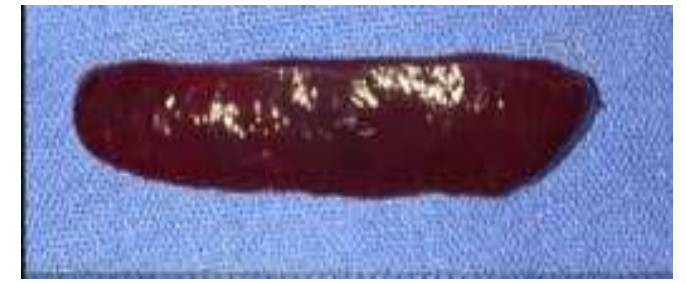

Disease control

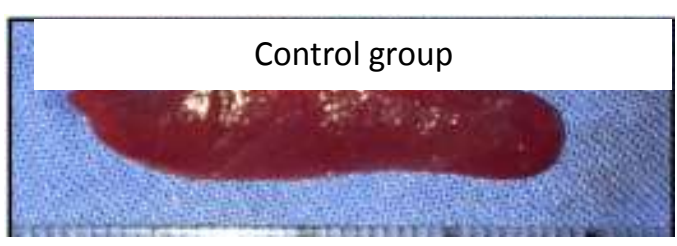

Test group

\section{Effect on rate of blood flow in Cobalt containing milk Inducing Polycythemia Rubra Vera:}

In this model, the animal was subjected for retro orbital blood collection and quantity of blood collected in 30 seconds were determined. The data were represented as Rate of Blood Flow in ml/minute. The study shown following results: 


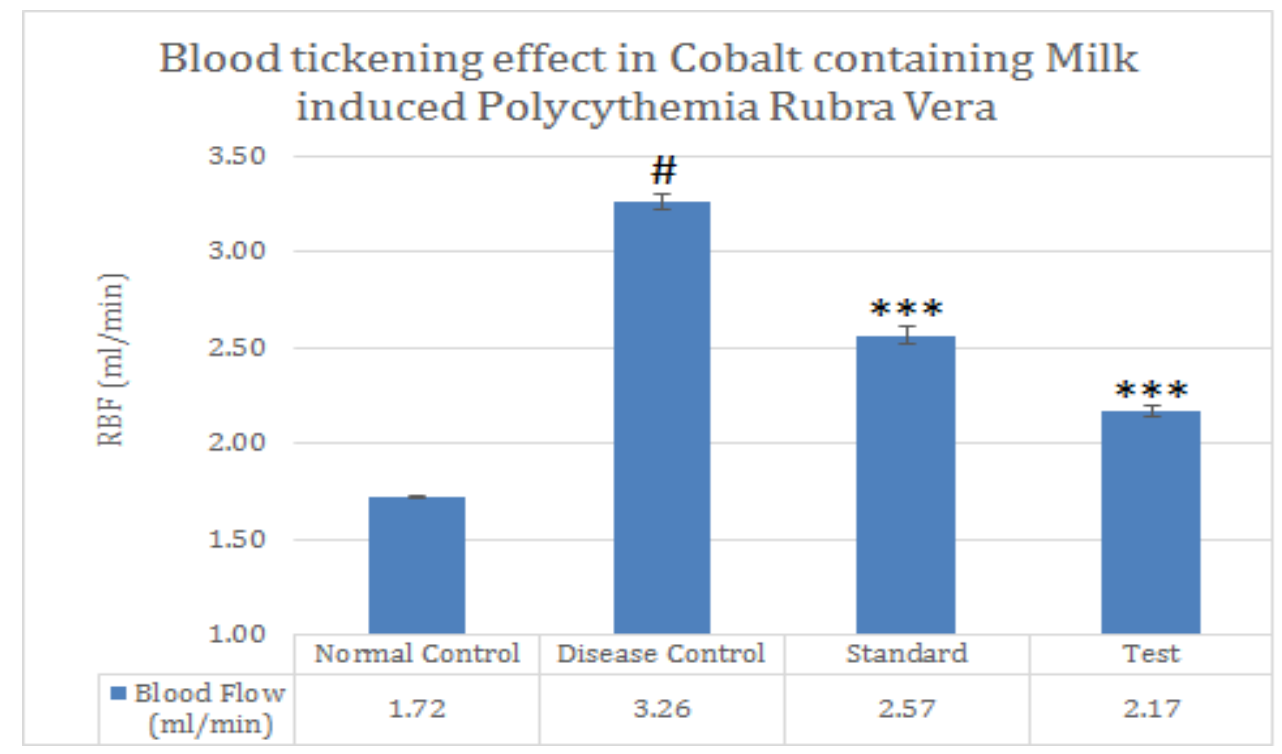

Fig 17 Effect on blood thickening in Cobalt containing milk inducing Polycythemia Rubra Vera

The study also shown that there was significant decrease in flow rate of blood in Disease control group (1.72 $\pm 0.04)$ as compare to that of Normal control group $(3.26 \pm 0.007)$ which was found significantly higher in Standard control group $(2.57 \pm 0.04)$ and Test control $(2.17 \pm 0.02) \mathrm{h}$ to that of the Disease control group.

For all the models, Normal control was DMSO $1 \mathrm{mg} / \mathrm{ml}$, Disease control was Cobalt containing milk diet $1 \mathrm{ml}$, Standard was Methotrexate $1 \mathrm{mg} / \mathrm{ml}$ and Test was Brinzolamide $1 \mathrm{mg} / \mathrm{ml}$.

\# indicate significant difference in Disease control and Normal control. *** indicate significant difference in Standard, Test and Normal control.

\section{Discussion:}

Polycythemia Vera (PV), essential thrombocythemia (ET) and myeloid metaplasia with myelofibrosis (MMM) are clonal disorders arising from hematopoietic progenitors. It's also says that it is an abnormal cell growth of Red Blood Cells. These mutant kinases have proven to be good candidates for molecularly targeted therapy. ${ }^{[4]}$ Phlebotomy is one form of treatment. In this removal of blood from the body induce iron deficiency, thereby decreasing the hemoglobin and hematocrit level and reducing the risk of blood clots. Other treatments include Chemotherapy, Stem cell transplantation and Radiation Therapy. To ensure therapeutic effectiveness of Brinzolamide in polycythemia rubra vera, we focused to work on animal model in which $\mathrm{RBC}$ cancer was induced by feeding Cobalt containing Milk diet to rat. It is believed that such diet on longer term exposure produces Oxygen deprivation in blood resulting into increased RBC generation and produces transient Polycythemia rubra vera like condition. In this model, polycythemia like condition was characterized by significant rise in RBC count, WBC Count, PT count, Hb, Hematocrit, MCH, MCHC, RDW, etc.

The study clearly shown beneficial effect of Brinzolamide on blood cell count and other relevant blood parameters viz., in disease control group all Blood cell count \& other parameters increased (except blood flow which was decreased) while there was significant reduction in blood cells count as increase in blood flow compared to that of Diseases control group. Here the level of significance was $\boldsymbol{P}<\boldsymbol{0 . 0 0 1}$. 


\section{Conclusion:}

The study carried out clearly revealed that brinzolamide has strong anti-cancer activity. This finding was supported by Animal study revealed cytotoxic potential - anticancer activity of brinzolamide in Cobalt containing milk induced polycythemia rubra vera model.

From the above findings we can conclude that Brinzolamide has strong anti-cancer potential specifically in Polycythemia rubra vera and can subjected for further study to know molecular mechanism of action of brinzolamide as an anti-cancer agent.

\section{References:}

1. Cancer Treatment Centers of America. Available at https://www.cancercenter.com/terms/blood.cancers/ (Accessed on - 22-08-2017)

2. American Society of Hematology Helping Hematologists conquer blood diseases worldwide available at - https://www.hematology.org/patients/cancers/ (Accessed on - 22-08-2017)

3. Cancer health center, Leukemia Center, Leukemia, available at https://www.medicinenet.com/leukemia/article.htm (Accessed on - 23-08-2017)

4. Levine, R. L., Wadleigh, M., Cools, J., Ebert, B. L., Wernig, G., Huntly, B. J. P., \& Gilliland, D. G. (2005). Activating mutation in the tyrosine kinase JAK 2 in Polycythemia Vera, essential thrmbocythemia, and myeloid metaplasia with myelofibrosis. Cancer Cell, 7(April), 387-397. doi:10.1016/j.ccr.2005.03.023.

5. John W. Adamson et al. (2005). Polycythemia Vera: Stem - cell and probable clonal origin of the Disease. The New England Journal Of Medicine, 295, 913-916

6. Polycythemia Vera Pathology, encyclopedia Britannica, available at https://www.britannica.com/science/polycythemia-vera (Accessed on - 24-08-2017)

7. Vera, P. Sign and Symptoms, available at - https://en.wikipedia.org/wiki/polycythemiavera\#cite_note-2 (Accessed on - 24-08-2017)

8. Komal S. Saini et al. (2010). Polycythemia Vera - Associated pruritus and its management, European journal of clinical investigation, 1365, 2362-2010.

9. Robbins basic pathology, 9th edition, By Vinay Kumar et al. Elsevier, Available at https://www.us.elsevier-health.com/robbins-basic-pathology-9781437717815.html (Accessed on - 2508-2017)

10. Patient care and Health information disease and condition, polycythemia disease and condition, Polycythemia Vera, Diagnosis and Treatment, Myoclonic, Available at https://www.mayoclonic.org/disease-condition/polycythemia-vera/home/ovc-20307463\#! (Accessed on - 25-08-2017)

11. Strieff, M. B., (2002). The diagnosis and management of polycythemia vera in the era since the Polycythemia Vera Study Group: a survey of American Society of Hematology members' practice patterns. Blood, 99(4), 1144-1149. doi:10.1182/blood.V99.4.1144.

12. Zhe li et al. (2007). Erlotinib effectively inhibit JAK2V617F activity and Polycythemia Vera cell growth" US International library of medicine and national institute of health, hhs public access, Journal of Biochemistry, 28(6), 3428-3432.

13. Open level Ruxolitinib (INCB018424) in patients with Myelofibrosis and post Polycythemia Myelofibrosis, a service of the US national institute of health, Available at - https://clinicaltrials,gov/ct2/show/NCT00509899 (Accessed on - 25-08-2017)

14. Nobuharu noma et al. (2017). Impact of Acetazolamide, a Carbonic Anhydrase inhibitor, on the development of intestinal polyps in Min Mice, International Journal of Molecular Science, 14(4), 851

15. Hatching Brine Shrimp cyst, Available at - https://www.brineshrimpdirect.com/c90/hatching-brineshrimp-cysts-c169.html (Accessed on - 25-08-2017)

16. Bonapace, G., Iuliano, F., Molica, S., Peta, A., \& Strisciuglio, P., \& the Giuseppe Bonapace et al. (2004). Cystosolic carbonic anhydrase activity in chronic myeloid disorder with different clinical phenotype. Biochimica et Biophysica Acta (BBA) - Molecular Basis of Disease, 1689(3), 179-181. doi:10.1016/j.bbadis.2004.05.003. 
17. Khan, S. A., (2005). Biological activites of Sulphonamides. Indian Journal of Pharmaceutical Sciences, 67(2), 151-159.

18. Orten, J. M., (1932). Polycythemia in the rat on a milk - iron - copper diet supplemented by Cobalt. The Journal of Biological Chemistry, 96, 11-28.

19. Bonapace, G., Iuliano, F., Molica, S., Peta, A., \& Strisciuglio, P. (2004). Cystosolic carbonic anhydrase activity in chronic myeloid disorder with different clinical phenotype. Biochimica et Biophysica Acta (BBA) - Molecular Basis of Disease, 1689(3), 179-181. doi:10.1016/j.bbadis.2004.05.003.

20. Polycythemia Vera Workup. Medscape, Available at - https://emedicine.medscape.com/article/205114workup (Accessed on - 19-12-2017)

21. Kwaan, H. C., \& Wang, J. (2003). Hyperviscosity in polycythemia vera and other red cell abnormalities. Seminars in Thrombosis and Hemostasis, 29(5), 451-458. doi:10.1055/s-2003-44552. 